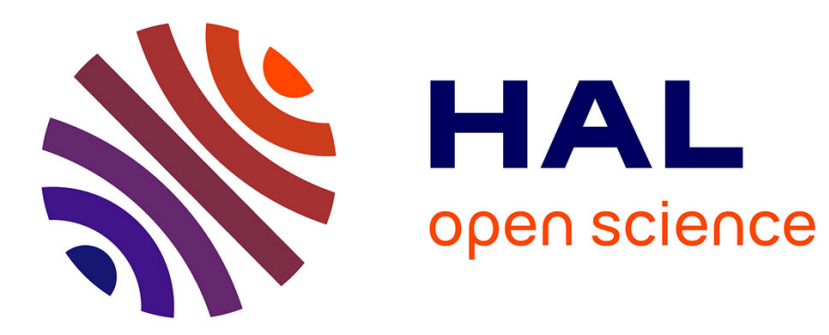

\title{
Origine et nature de la contamination involontaire des couches de silicium épitaxique sur substrat corindon $(\mathrm{Al} 2 \mathrm{o} 3-\alpha)$
}

\author{
J. Mercier
}

\section{- To cite this version:}

J. Mercier. Origine et nature de la contamination involontaire des couches de silicium épitaxique sur substrat corindon (Al2o3- $\alpha$ ). Revue de Physique Appliquée, 1968, 3 (2), pp.127-130. 10.1051/rphysap:0196800302012700 . jpa-00242836

\section{HAL Id: jpa-00242836 https://hal.science/jpa-00242836}

Submitted on 1 Jan 1968

HAL is a multi-disciplinary open access archive for the deposit and dissemination of scientific research documents, whether they are published or not. The documents may come from teaching and research institutions in France or abroad, or from public or private research centers.
L'archive ouverte pluridisciplinaire HAL, est destinée au dépôt et à la diffusion de documents scientifiques de niveau recherche, publiés ou non, émanant des établissements d'enseignement et de recherche français ou étrangers, des laboratoires publics ou privés. 


\title{
ORIGINE ET NATURE DE LA GONTAMINATION INVOLONTAIRE DES GOUGHES DE SILIGIUM ÉPITAXIQUE SUR SUBSTRAT GORINDON $\left(\mathrm{Al}_{2} \mathrm{O}_{3}-\alpha\right)$
}

\author{
Par J. MERGIER, \\ C.N.R.S., Grenoble. \\ (Reçu le 3 novembre 1967.)
}

\begin{abstract}
Résumé. - Une méthode purement électrique permet de déterminer qualitativement et quantitativement les sources de contamination de type $p$ que l'on observe effectivement sur les couches épitaxiques de silicium sur $\mathrm{Al}_{2} \mathrm{O}_{3}-\alpha$ par pyrolyse du silane. L'aluminium est reconnu comme l'agent dopant, et les causes de contamination sont, d'une part, le suscepteur, d'autre part et surtout, le substrat d'alumine, par un mécanisme incertain. Une réduction considérable du taux de contamination par l'emploi d'une protection en quartz ne peut s'expliquer que par un effet actif de piégeage du dopant.
\end{abstract}

Abstract. - A purely electrical method permits qualitative and quantitative determination of the sources of $p$-type contamination, which are actually observed in the epitaxial silicon layers on sapphire, produced by pyrolysis of silane. Aluminium is claimed to be the doping agent and the origins of contamination are from one side, the susceptor, from the other side and mainly the alumina substrate, by an uncertain mechanism. A considerable reduction of the contamination level by mean of a quartz protection can only be explained by a gettering effect on the dopant.

Introduction. - L'étude du dépôt épitaxique de silicium sur saphir entreprise dans notre Laboratoire par M. J.-L. Fraimbault [1] montre qu'une contamination de type $p$ marqué, non intentionnelle, des couches déposées, est systématiquement observée. J. D. Filby [2] et J.-L. Fraimbault l'interprètent comme le résultat d'une réaction $\mathrm{Al}_{2} \mathrm{O}_{3}-\mathrm{Si}$, en présence d'hydrogène, à haute température, lors du décapage thermique du substrat.

Il a effectué des mesures en microsonde de Castaing pour déterminer la nature du contaminant et met ainsi en évidence l'aluminium. Les seuls doutes qui subsistent portent sur l'unicité de l'origine de cette contamination et la quantité d'Al donnée par la sonde, de l'ordre de 500 p.p.m. ( 6 à $10 \times 10^{19}$ atomes $\left./ \mathrm{cm}^{3}\right)$ qui ne correspond pas à l'ordre de grandeur des concentrations électriquement mesurées $(0,1$ à $1 \Omega \mathrm{cm}$ sans précaution) sur les couches épitaxiques.

De plus, des mesures électriques effectuées au Laboratoire de la C.S.F. (Recherches Physico-Chimiques) sur des couches $\mathrm{Si} / \mathrm{Al}_{2} \mathrm{O}_{3}$ élaborées sous ultra-vide au Laboratoire de la R.T.C. à Caen (M. Strepkoff [3]) font apparaître des anomalies de ces couches.

Il s'avère donc nécessaire d'assurer une meilleure compréhension et si possible une réduction de ce phénomène de contamination non intentionnelle.
Méthode expérimentale. - Les couches sont élaborées par pyrolyse du silane à haute température, en présence d'hydrogène dans une chambre à réaction actuellement verticale.

Nous avons cherché à analyser cet effet de la manière la plus simple possible, mais également la plus significative, en opérant dans les conditions proches de celles usuellement adoptées (débits et températures en particulier). Pour cela, nous avons employé des combinaisons variées d'empilements : suscepteurintercalaire-substrat $\mathrm{Al}_{2} \mathrm{O}_{3}$-plaquette témoin en silicium de type $n$, de résistivité connue.

Le débit d'hydrogène principal est normalement $d_{\mathrm{H}_{2}}=14 \mathrm{l} / \mathrm{mn}$.

Les substrats $\mathrm{Al}_{2} \mathrm{O}_{3}$ subissent le traitement habituel de polissage et de nettoyage, avant introduction dans la chambre.

Les températures indiquées sont corrigées (étalonnage par point de fusion de l'or).

Le suscepteur est un bloc cylindrique de graphite enrobé par SiG. L'enrobage est effectué soit à l'extérieur, soit au Laboratoire, selon une méthode qui nous a été gracieusement donnée par la R.T.C.

La méthode de contrôle est du type électrique : mesures de la résistivité de couche inversée (s'il y a lieu) sur les témoins de type $n$ initialement, et révé- 
lation (réactif de Dash) de l'épaisseur de couche inversée $x_{\mathrm{j}}$. On admet que la distribution suit une loi en erfc et on utilise les classiques abaques d'Irvin [4] pour en déduire la concentration superficielle $C_{\mathrm{s}}$ de dopant. Connaissant $C_{\mathrm{s}}$ et $x_{\mathrm{j}}$ et la loi de répartition d'impuretés, on en déduit la constante de diffusion $D$ à la température considérée. Toute la valeur des conclusions tirées de cette procédure est issue de la remarque suivante : quelles que soient les conditions expérimentales et les valeurs publiées de la constante de diffusion, celle-ci est de l'ordre de dix fois plus grande (à $T$ donnée) pour $\mathrm{Al}$ que pour le bore et le gallium, dans le cas du silicium [5], [6], [7]. De plus, il est connu [8] que les cristaux de $\mathrm{SiC}$ renferment comme impuretés principales $\mathrm{N}_{2}$ et $\mathrm{Al}$, ce qui nous a conduit à examiner la contribution du suscepteur enrobé.
Résultats expérimentaux. - Ces résultats sont groupés dans un tableau comportant avec le numéro d'essai, référé sur la figure 3 :

$1^{0}$ La nature de l'empilement qui est une combinaison de :

- plaquette de silicium témoin : $\mathrm{Si}$,

- plaquette substrat d'alumine- $\alpha: \mathrm{Al}_{2} \mathrm{O}_{3}$,

- enveloppe intercalaire de quartz : $\mathrm{SiO}_{2}$,

- enrobage du suscepteur : $\mathrm{SiC}$ éventuellement recouvert au préalable du silicium à partir de $\mathrm{SiH}_{4}$

$2^{\circ}$ Les températures de surface relevées au pyromètre optique et corrigées par étalonnage, sur les divers étages de l'empilement;

$3^{0}$ La résistivité du silicium témoin (de type $n$ );

\begin{tabular}{|c|c|c|c|c|c|c|c|c|c|c|}
\hline $\mathrm{N}^{\mathrm{os}}$ & EMPILEMENT & $T^{\circ} \mathrm{C}$ & $\begin{array}{c}P \\
\text { TÉMOIN } \\
\Omega \mathrm{cm}\end{array}$ & $\begin{array}{c}\begin{array}{c}C_{\mathrm{B}} \\
\mathrm{cm}^{-3}\end{array}\end{array}$ & $\begin{array}{l}\text { INVERSION } \\
\text { TÉMOIN }\end{array}$ & $\begin{array}{l}x_{\mathrm{j}}(\mu) \\
(\text { fig. } 1)\end{array}$ & $\begin{array}{l}\sigma \\
\Omega^{-1} \mathrm{~cm}^{-1}\end{array}$ & $\begin{array}{c}C_{\mathrm{s}} \\
\mathrm{cm}^{-3}\end{array}$ & $\begin{array}{l}\lambda D \\
\mu\end{array}$ & $\begin{array}{c}D \\
\mathrm{~cm}^{2} / \mathrm{s}\end{array}$ \\
\hline \multirow[t]{2}{*}{-} & - & - & - & - & 一 & 一 & - & - & - & - \\
\hline & $\mathrm{Si}$ & 1220 & & & oui & & & & & \\
\hline \multirow[t]{3}{*}{1} & $\mathrm{Al}_{2} \mathrm{O}_{3}$ & & 3,5 & $1,5 \times 10^{15}$ & & 8,65 & 32 & $1,5 \times 10^{19}$ & 3,1 & $2 \times 10^{-11}$ \\
\hline & $\mathrm{SiC}$ & 1330 & & & 2 faces & & & & & \\
\hline & $\mathrm{Si}$ & 1060 & & & non & & & & & \\
\hline \multirow{4}{*}{2} & $\mathrm{Al}_{2} \mathrm{O}_{3}$ & 1220 & 3,5 & $1,5 \times 10^{15}$ & $\rho$ est & Résultat q & antitativem & ent difficile à & xpliquer & (effet d'O $\mathrm{O}_{2}$ ?). \\
\hline & inter $\mathrm{SiO}_{2}$ & 1280 & & & devenue & & & & & \\
\hline & $\mathrm{SiC}$ & & & & $2,4 \Omega \mathrm{cm}$ & & & & & \\
\hline & $\mathrm{Si}$ & 1210 & & & oui & oui & & & & \\
\hline \multirow[t]{4}{*}{3} & $\mathrm{Al}_{2} \mathrm{O}_{3}$ & 1230 & 3,5 & $1,5 \times 10^{15}$ & 2 faces & 1 face & & & & \\
\hline & $\mathrm{SiC}$ & 1350 & & & & en regard & & & & \\
\hline & & & & & & $\mathrm{Al}_{2} \mathrm{O}_{3}$ & & & & \\
\hline & $\mathrm{Si}$ & 1080 & & & oui & & & & & \\
\hline \multirow[t]{3}{*}{4} & $\mathrm{Al}_{2} \mathrm{O}_{3}$ & & 4,5 & $10^{15}$ & 1 face & 1,7 & 10 & $3 \times 10^{18}$ & 0,7 & $6,2 \times 10^{-12}$ \\
\hline & $\mathrm{SiC}$ & 1140 & & & en regard & & & & & \\
\hline & & & & & $\mathrm{Al}_{2} \mathrm{O}_{3}$ & & & & & \\
\hline \multirow[t]{2}{*}{5} & $\mathrm{Si}$ & 1215 & 4,5 & $10^{15}$ & oui & 8,9 & 4 & $6 \times 10^{17}$ & 3,95 & $3,2 \times 10^{-11}$ \\
\hline & $\mathrm{SiC}$ & 1320 & & & 2 faces & & & & & \\
\hline \multirow{3}{*}{6} & $\mathrm{Si}$ & 1070 & & & non & & & & & \\
\hline & inter $\mathrm{SiO}_{2}$ & 1230 & 4,5 & $10^{15}$ & \multirow{2}{*}{\multicolumn{6}{|c|}{$\begin{array}{l}\text { La résistivité est passée de } 4,5 \Omega \mathrm{cm} \text { à } 70 \Omega \mathrm{cm} \text { : on peut l'expliquer par une } \\
\text { compensation équivalant à } 15 \Omega \mathrm{cm} / \mathrm{p} \text {. }\end{array}$}} \\
\hline & $\mathrm{SiC}$ & 1310 & & & & & & & & \\
\hline \multirow{3}{*}{7} & $\mathrm{Si}$ & 1140 & & & oui & & & & & \\
\hline & $\begin{array}{l}\text { SiC recouvert } \\
\text { de } \mathrm{Si}\end{array}$ & 1240 & 4,5 & $10^{15}$ & 2 faces & 6,8 & 1,7 & $1,5 \times 10^{17}$ & 3,5 & $1,7 \times 10^{-12}$ \\
\hline & $\mathrm{Si}$ & 1150 & & & & & & & & \\
\hline \multirow[t]{2}{*}{8} & $\mathrm{Al}_{2} \mathrm{O}_{3}$ & 1220 & 4,5 & $10^{15}$ & oui & 5,2 & 17,5 & $7 \times 10^{17}$ & 2,3 & $7,4 \times 10^{-11}$ \\
\hline & $\mathrm{SiC}$ recouvert & 1300 & & & 2 faces & & & & & \\
\hline
\end{tabular}


4o La concentration $C_{\mathrm{B}}$ en accepteurs correspondant à cette résistivité;

$5^{\circ}$ L'indication du type en surface sur le témoin, après traitement thermique;

$6^{\circ}$ L'épaisseur de couche inversée déterminée par attaque au Dash-Etch sur un biseau de $3^{\circ}$ et dont un exemple est donné par la figure 1;

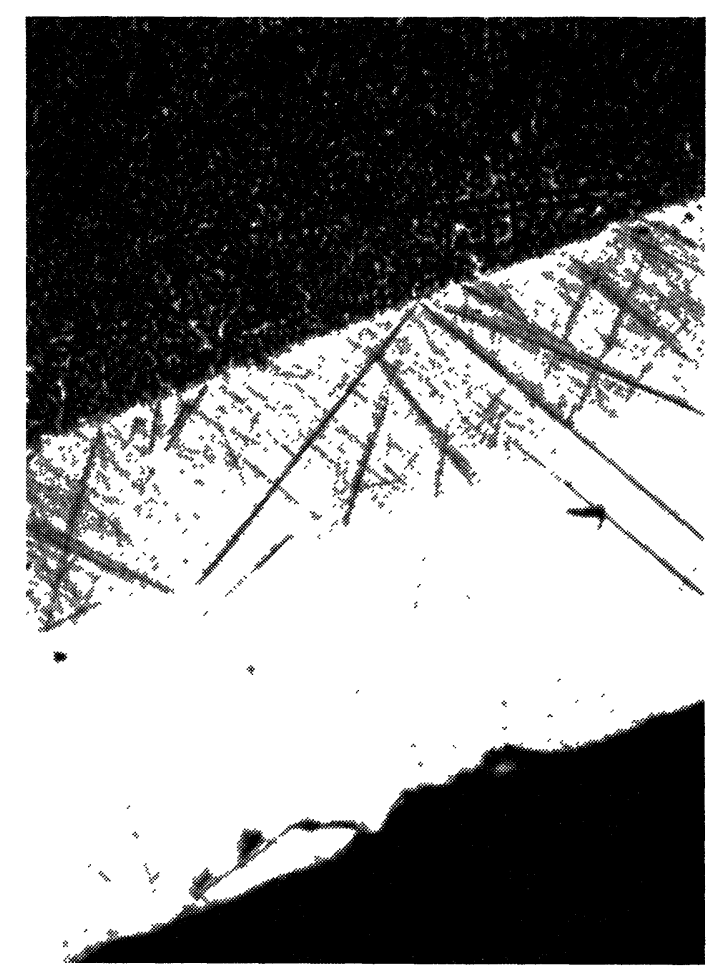

FIG. 1. - Révélation de zone inversée. Biseau de $3^{\circ}$ vers le haut. Grossissement $140 \times$.

$7^{0}$ La conductivité moyenne de la couche déterminée à partir de la mesure de résistance de couche aux quatre pointes.

D'après les abaques d'Irvin, supposant connue la loi de distribution d'impuretés - à savoir une erfc on peut calculer la concentration superficielle $C_{\mathrm{s}}$, et avec la valeur de $C_{\mathrm{B}}$ on obtient la longueur caractéristique de diffusion :

$$
\lambda_{\mathrm{D}}=2 \sqrt{\overline{D t}}
$$

$t$ : temps de traitement; $D(T)$ : constante de diffusion caractéristique de l'impureté dopante.

Interprétation. - 1) Sur les ordres de grandeur de contamination, on retrouve en surface des concentrations très élevées, quand on opère à haute température, en présence d' $\mathrm{Al}_{2} \mathrm{O}_{3}$ et de suscepteur enrobé $\mathrm{SiC}$ seul (essais 1 et 3 ).

2) La contamination diminue cependant beaucoup quand, dans les mêmes conditions opératoires, la température s'abaisse (essai 4).
3) L'inversion de surface est supprimée dans le cas où l'on emploie un intercalaire en quartz qui recouvre complètement le suscepteur. Néanmoins, en présence d'alumine, le résultat (essai 2) s'interprète difficilement quantitativement. Nous pensons que ces changements de résistivité doivent être associés à la présence d'oxygène libéré par l'attaque de l'alumine, qui peut donner des états donneurs [9]. Dans le cas du couple $\mathrm{Si}-\mathrm{SiC}$, on trouve une compensation simple.

4) Par comparaison de l'essai 5 et des essais 1 et 3 , on peut attribuer la majeure partie de la contamination à la présence de l'aluminium provenant de

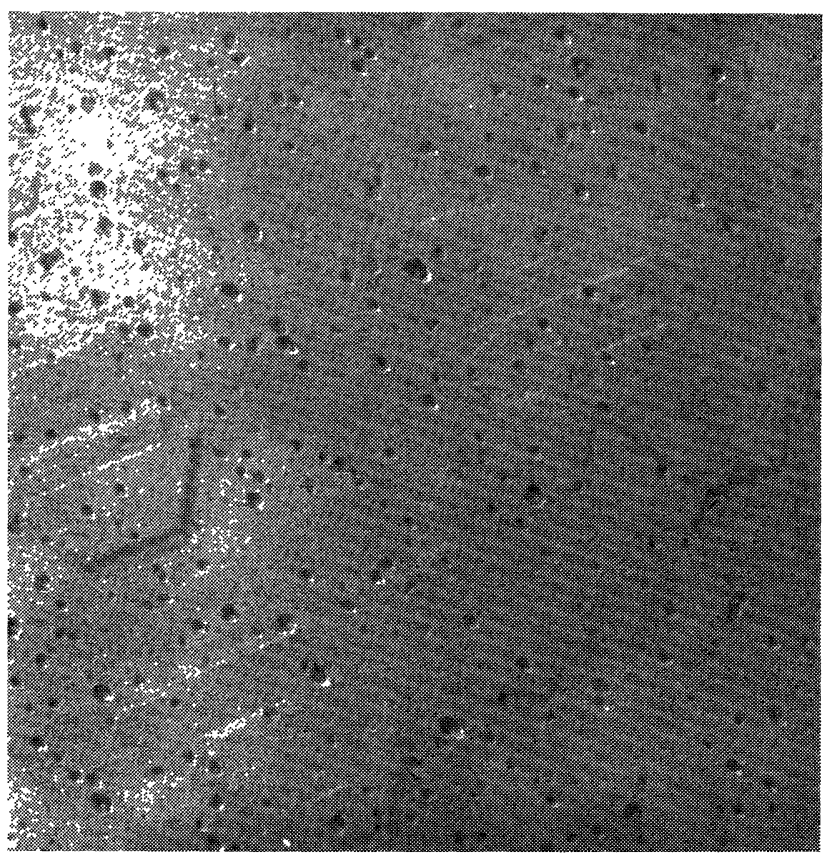

FIG. 2. - Figures d'attaque thermique du corindon sous hydrogène. Grossissement $20000 \times$.

l'attaque du substrat (voir cliché fig. 2) : la concentration $C_{\mathrm{s}}$ baisse en effet de presque deux ordres de grandeur.

5) La comparaison des essais 6 et 7 indique que la protection par couche de silicium déposée est un peu moins efficace que celle fournie par l'intercalaire de quartz, mais que la contamination est moindre qu'avec l'enrobage SiC.

6) Enfin, dans des conditions intermédiaires de température et de protection (partie du suscepteur enrobée de $\mathrm{Si}$ ), la contamination prend des valeurs intermédiaires.

Conclusion : L'adoption dans la mesure du possible d'un intercalaire totalement enrobant de $\mathrm{SiO}_{2}$ réduit la contamination à des proportions raisonnables. Il faut noter que son origine est double : l'attaque de 
l'alumine en majeure partie, le suscepteur en moindre proportion. Ce résultat est confirmé par les valeurs de résistivité obtenues sur des dépôts épitaxiques qui excèdent $10 \Omega \mathrm{cm} / \mathrm{p}$ quand on utilise cette protection.

Nature du contaminant. - Nous avons porté sur un graphique les courbes $D_{\text {diffusion }}$ de l'aluminium et du bore dans le silicium; d'après les références citées [7 et 6] et les points expérimentaux ( fig. 3), il est clair que seul l'aluminium répond à ces valeurs de constante de diffusion.

Conclusion : Les essais présentés permettent d'affirmer la présence d'une contamination des couches par l'aluminium. Une solution est proposée pour réduire ce taux de contamination à une valeur raisonnable. Néanmoins, il n'est pas évident que le rôle de l'enveloppe de quartz soit purement celui d'une protection mécanique : nous penchons plutôt vers le rôle actif de « getter » d'aluminium, compatible avec son coefficient de partage que l'on sait être favorable devant celui du silicium. Ceci permettrait d'expliquer pourquoi les résistivités de couches épitaxiques de silicium sur corindon sont nettement plus élevées que celles que l'on déduirait de ces mesures (ainsi que celles données par la sonde de Castaing). Il reste cependant que l'épitaxie par substitution des atomes $\mathrm{Al}$ par des atomes $\mathrm{Si}$ avec constitution de liaison $\mathrm{Si}-\mathrm{O}$, peut conduire à d'autres défauts cristallins que nous étudions actuellement.

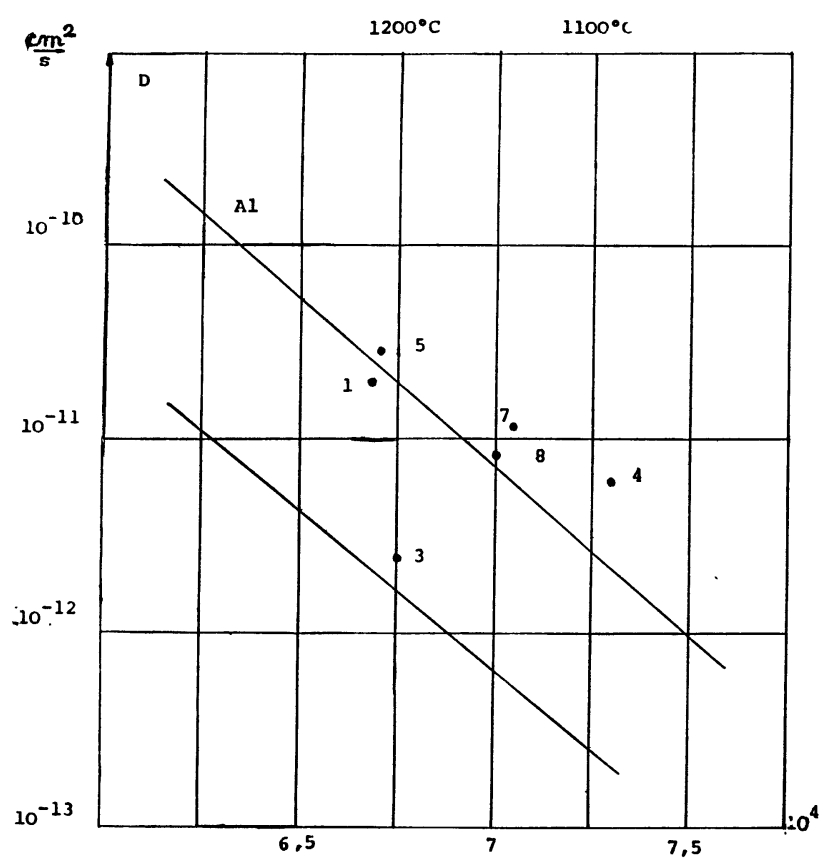

FIG. 3. - Variation de la constante de diffusion d'impuretés de type $p$ dans le silicium, en fonction de la température. Comparaison avec les valeurs expérimentales : essais $\mathrm{n}^{\mathrm{os}} 1,3,4,5,7,8$.

Erratum. - La droite inférieure correspond au bore.

\section{BIBLIOGRAPHIE}

[1] Fraimbaulit (J.-L.), Thèse à 1'Université de Grenoble, 12-7-67.

[2] FILBy (J. D.), J. of Electrochem. Soc., 1966, 113, 10, 1085-1087.

[3] Réunion D.R.M.E., 29 juin 1966, Exposés de MM. Strepkoff (R.T.C.) et Jund (C.S.F.).

[4] Irvin (J. C.), B.S.T.J., mars 1962, 41, 387-410.
[5] KuRTz et YEe, J. Appl. Phys., février 1960, 31, 303-305.

[6] KuRTz et Gravel, J. Appl. Phys., 1958, 29, 1456-1459.

[7] Mili,er et Savage, $J$. Appl. Phys., décembre 1956, 27, 1430-1432.

[8] Slack (G. A.), J. Appl. Phys., décembre 1964, 35, 3460-3466.

[9] KAISER-KECK, J. Appl. Phys., août 1957, 28, 882-887. 